\title{
Nucleation, growth and transformation of microdefects in FZ-Si
}

\author{
V.I. Talanin, I.E. Talanin* \\ Zaporozhye Institute of State \& Municipal Government, 70B, Zhukovskii str., 69002 Zaporozhye, Ukraine \\ E-mail:V.I.Talanin@mail.ru \\ *Zaporozhye State Engineering Academy, 226, prospect Lenina, 69006 Zaporozhye, Ukraine \\ Fax: $+380(612) 601498$
}

\begin{abstract}
The physical model of microdefects formation in dislocation-free FZ-Si single crystals is offered. Experimental results and theoretical data allows to approve that recombination between vacancy and self-interstitials at high temperatures is hampered by an entropy barrier. Established is that the process of microdefects formation in silicon proceeds simultaneously by two independent mechanisms: the vacancy and interstitial ones.
\end{abstract}

Keywords: microdefects, silicon, interstitial, vacancy.

Paper received 29.11.03; accepted for publication 30.03.04.

\section{Introduction}

During the production of dislocation-free silicon single crystals, it is necessary to solve the problem of their structural perfection. After the crystals are grown, structural microdefects form during cooling, which may include agglomerates of point defects (vacancies or silicon selfinterstitials) and impurities. These structural defects can detrimentally affect the reliability of semiconductor devices and their performances.

The systematic study of microdefects began in 1960s, using methods of selective etching, decoration and X-ray topography [1-3]. On the basis of these studies, two types of microdefects were identified: A-microdefects (usually revealed as large etch-pits with smaller concentration) and $B$-microdefects (small etch-pits with higher concentration) [4]. Experience of crystal quenching [5] has shown that $B$-microdefects are formed first. Using transmission electronic microscopy (TEM) it was established that $A$-microdefects [6] and $B$-microdefects [7, 8] have an interstitial character. Typical $A$-microdefects are observed in stratified distribution at crystal growth rates of $V=1 \ldots 3.5 \mathrm{~mm} / \mathrm{min}^{-1}$, and in uniform distribution at $V<1 \mathrm{~mm} / \mathrm{min}^{-1}$. B-microdefects are observed in stratified distribution at $V \leq 4.5 \mathrm{~mm} \mathrm{~min}^{-1}$. Such a distribution of microdefects reflects the distribution of their nucleation sites. During crystal growth, there is a fluctuation in the temperature due to the rotation of the crystal and a melt convection occurs [9]. Therefore, the microdefects grow, repeating crystal growth and stopping growth [10].

Further studies of silicon single crystals grown at high growth rates (more than $4.5 \mathrm{~mm} / \mathrm{min}^{-1}$ ) have shown that these monocrystals contain uniformly distributed microdefects; this was revealed under selective etching as matt areas. Veselovskaya et al. [11] observed these defects in FZ-Si by selective etching and decoration techniques, and classified them as $C$ - and $D$-microdefects depending on their distribution and concentration. Both these types of microdefects were found as areas of uniformly distributed defects with high densities. The difference between $C$ and $D$-defects is in the distribution of microdefects in these areas. $D$-microdefects are primarily concentrated as channels in a central part of the crystal whereas $C$-microdefects are revealed as rings or contours of an incorrect form. Later, Roksnoer et al. [12], using X-ray topography followed by decoration with copper, suggested that $D$-microdefects have a vacancy characteristic in Czochralski grown $(\mathrm{Cz}-\mathrm{Si})$ silicon.

Various theoretical models were suggested to explain the regularities of formation of microdefects in silicon. Main problems were in the assumptions about the dominant type of point defects in crystal, their concentration, and interaction between them. In some models $[6,10$, 13], it was assumed that the dominating type of point defects in crystal are self-interstitials. In other models $[14,15]$, it was supposed that the dominating type of defects are the vacancies. In contrast, authors of Refs [16, 


\section{V.I. Talanin, I.E. Talanin: Nucleation, growth and transformation of microdefects in FZ-Si}

17] suggested simultaneous independent coexistence of both main types of point defects at high temperatures. However, none of these models could explain experimental results, which were obtained later with TEM [18].

According to the commonly accepted Voronkov theory [19-21], the recombination rate between isolated vacancy and interstitial defects is high and further the diffusivity of interstitials is higher than the diffusivity of vacancies near the melting point, and finally the concentrations of vacancies is higher than the concentrations of interstitials at the melting point where both concentrations are in thermal equilibrium. Only the microdefects of either interstitial type ( $A$ - and $B$-microdefects, if the concentration of self-interstitials is higher than the concentration of vacancies) or only the vacancy type ( $D$-microdefects, if the concentration of vacancies is higher than the concentration of interstitial atoms) are formed in the crystal. According to [19], the type of dominating point defects depends on the parameter $V / G(V-$ growth rate of crystal, $G$ - axial temperature gradient): if $V / G<C_{\text {crit }}$, then interstitial atoms of silicon dominate in the crystal, if $V / G>C_{c r i t}$, then the vacancies dominate. Furthermore, these results based on data from Refs. [10, 12].

Thus, the sense of the Voronkov model consists of the following: a) existence of recombination between selfinterstitials and vacancies for temperatures close to the temperature of smelting; b) supposed was the only vacancy nature for primary grown-in microdefects; c) independent existence of areas with only interstitial and only vacancy microdefects (see Fig. 1).

\section{Experimental methods}

Non-doped monocrystals of high resistivity (2200... ..4000 cm) $n$-type silicon by diameter of $30 \mathrm{~mm}$ were grown by floating zone technique in vacuum. The number of passes of a melting zone varied from 2 up to 10 . The concentration of oxygen and carbon, defined by IR-absorption was less than $5 \cdot 10^{15} \mathrm{~cm}^{-3}$. The crystals received at a constant growth rate in a range $1 . .9 \mathrm{~mm} / \mathrm{min}^{-1}$. Some

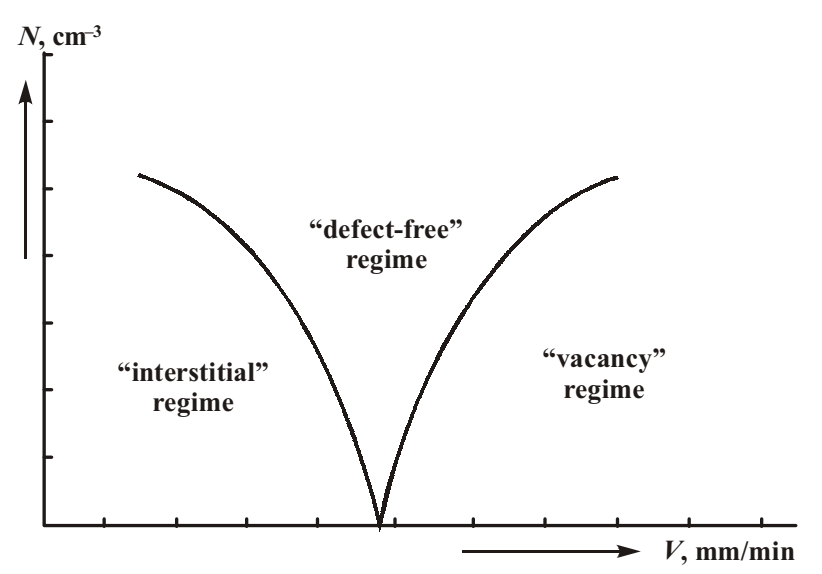

Fig. 1. Defect formation scheme according to the Voronkov theoretical model. crystals were obtained with modification of growth rate on fixed length.

In these crystals, if growth conditions are changed, we can observe all the types of microdefects. When the crystals diameter is increased, the growth condition are changed. As a result, in largescale crystals we can observe only some types of microdefects.

The method of selective etching of the cross-sections of crystal [22] with subsequent TEM-analysis was used to reveal the distribution of grown-in microdefects. The TEM studies were performed using the methods of the "black-white contrast"[23], 2,5D [24] and "inside-outside contrast"'[25]. Also applied were electron microscopes JEM-7A and TSEM-200 with accelerating voltage up to $100 \mathrm{kV}$. For such voltage the introduction of radiation defects is excluded, i.e. the quality of experiment is improved.

\section{Results and discussion}

Summarizing all the experimental results about the physical nature of microdefects in dislocation-free single crystal FZ-Si with a diameter of $30 \mathrm{~mm}$, then using classification [11] it is possible to conclude that:

1. $A$-microdefects are interstitial dislocation loops with sizes of $1-50 \mu \mathrm{m}$ with a Burgers vector of $\bar{b}=1 / 2$ [110], which are in planes $\{111\}$ and $\{110\}$.

2. $B$-microdefects are agglomerates of point defects of an interstitial type with sizes of $20-50 \mathrm{~nm}$, some of which are in the plane $\{100\}$. Using TEM these are represented as rectangles and rhombs in the plane $\{111\}$ with the parties on directions [110] and [100], respectively.

3. D-microdefects of an interstitial type are agglomerates of point defects with sizes of 4 to $10 \mathrm{~nm}$. Considering these as small dislocation loops, it is possible to conclude that they can be in planes $\{100\},\{110\}$, $\{111\}$ and have the Burgers vector $\bar{b}=1 / 2[100]$ and $\bar{b}=1 / 2$ [110].

4. $C$-microdefects are completely identical to $D$-microdefects in TEM images, sign of deformation of crystalline lattice and their sizes.

5. $D$-microdefects are uniformly distributed $B$-microdefects.

6. In crystals obtained at high growth rates (more than $6 \mathrm{~mm} / \mathrm{min}^{-1}$ ) microdefects of the vacancy type are formed simultaneously with microdefects of the interstitial type in the same regions of the crystal.

The experiments have shown that the nucleation and growth of microdefects depends on conditions of crystal growth (growth rate of crystal, cooling rate and temperature gradient) and on the concentration of impurities of carbon and oxygen. Therefore, it is possible to offer a qualitative model of formation, growth and transformation of microdefects in dislocation-free single crystals FZ-Si.

The analysis of literary data and the experiments in this work show a significant role of self-interstitials in high-temperature experiments. All the known types of 
microdefects in FZ-Si (i.e. $A$-, $B$-, $D$-defects) have an interstitial nature. Furthermore, in researches of a gold diffusion in silicon it was shown that the atoms of gold take substitutional $\left(\mathrm{Au}_{s}\right)$ and interstitial positions $\left(\mathrm{Au}_{i}\right)$ in silicon. The diffusion of gold in silicon is not explained using the Frank-Turnbull mechanism $\left(\mathrm{Au}_{i}+V \leftrightarrow \mathrm{Au}_{s}\right)$ [26], but logic explanation may be given in the kick-out mechanism [27]: $\mathrm{Au}_{i} \leftrightarrow \mathrm{Au}_{s}+I$.

Also we have the experimental results that the significant role is played by vacancies $[16,28]$. Thus, the correlation of thermodynamic accounts and experimental data is possible only in the model that suppose coexistence of self-interstitials and vacancies in a thermal equilibrium at high temperatures. In this model, the diffusivity of impurities is the sum of diffusivities on interstitials and on vacancies [28]. In Ref. [29] theoretical models of a selfdiffusion in silicon with simultaneous participation of self-interstitials and vacancies were offered. It was shown [29] that interstitial and vacancy contributions to a self-diffusion are equalized in the vicinity $11000 \mathrm{C}$, and for higher temperatures during a self-diffusion the selfinterstitials dominate whereas for a lower temperatures the vacancies dominate. The results obtained in Refs $[30,31]$ particularly confirm this model.

In our TEM-researches, it is established that vacancy microdefects occur together with interstitial microdefects in crystals grown at $V>6 \mathrm{~mm} / \mathrm{min}^{-1}$. A ratio of vacancy and interstitial microdefects in samples obtained at $V=$ $=7.5 \mathrm{~mm} / \mathrm{min}^{-1}$ is approximately $1: 4$. In crystals obtained at $V=9 \mathrm{~mm} / \mathrm{min}^{-1}$, interstitial and vacancy defects coexist approximately in the identical concentration, i.e. $1: 1$. Thus, the critical growth rate of the crystal for which vacancy microdefects occur is in the interval of $6 \mathrm{~mm} / \mathrm{min}^{-1}<V<6.5 \mathrm{~mm} / \mathrm{min}^{-1}$ (Fig. 2).

If the concentration of vacancy and interstitial microdefects are approximately identical, it is possible to assume that in equilibrium conditions vacancies and selfinterstitials exist simultaneously and also in approximately identical concentrations. The estimates from Ref. [19] give the concentration of vacancies at the smelting temperature are $1.5 \cdot 10^{14} \mathrm{~cm}^{-3}$, but selfinterstitials are $1.3 \cdot 10^{14} \mathrm{~cm}^{-3}$.

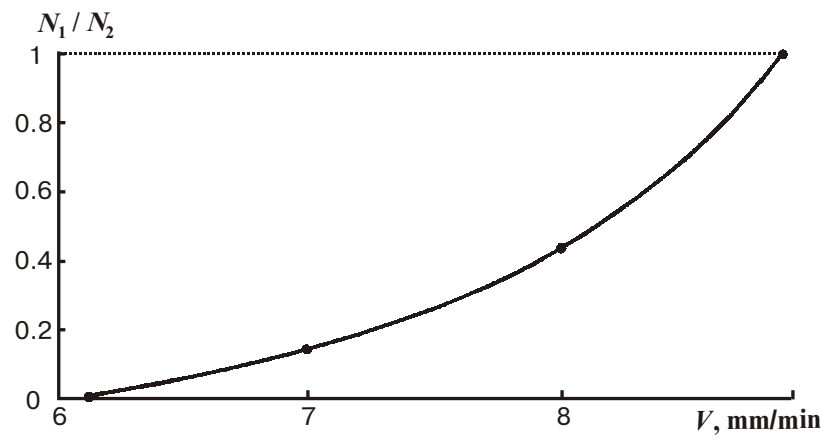

Fig. 2. Experimental dependence of the ratio of concentration of vacancy (N1) and interstitial (N2) microdefects on crystal growth rate.
In the Voronkov theory [19], it is suggested that the main role at the initial stage of disintegration of oversaturated solid solution of point defects is played by the process of a recombination between vacancies and self-interstitials.

However, in Refs $[16,32]$ it was supposed that the direct recombination between vacancies and selfinterstitials is hampered by the existence of an energy barrier. Tempelhoff et al. [32] argued that the recombination takes place only on some centres ( $B$ - and $A$-microdefects, dislocations, surface of crystal), and the direct recombination is impossible. In Refs $[17,33]$, it was discussed the theory of the energy barrier which is hampered by direct recombination between vacancies and selfinterstitials.

In the Ref. [17], the local equilibrium was considered

$C_{I} C_{V}=C_{I}^{e q v} C_{V}^{e q v}$

where $C_{V}$ is the concentration of vacancies; $C_{I}$ is the concentration of self-interstitials.

The order of values for times when the system achieves an equilibrium in the absence of a recombinational barrier can be calculated according to the formula:

$$
\tau \leq \frac{\Omega}{4 \pi D^{S} r_{0}},
$$

where $\Omega$ is the volume of an elementary cell; $D^{S}$ is the self-diffusion constant; $r_{0}$ is a recombination cut.

Estimates from Ref. [33] at $D^{S}=10^{-15} \mathrm{~cm}^{2} / \mathrm{s}, T=$ $=1100^{\circ} \mathrm{C}, r_{0}=5 \cdot 10^{-8} \mathrm{~cm}$ give the values $\tau \leq 0.05$. This value is $10^{5}$ less than the experimentally observed values [33]. Thus, the conclusion can be made that the recombination is determined not by a diffusion, but overcoming the recombinational barrier exceeding the free energy of diffusion by $\Delta G$. The value $10^{5}$ arises from the Boltzmann

factor $(\exp \Delta G / k T)$.

In Ref. [33], it was supposed that the recombinational barrier is the enthalpy barrier and entropy component $T \Delta S$ is very small:

$\Delta G=\Delta H-T \Delta S$

However, in Ref. [34] remarked was that the intrinsic point defects behave variously for the various temperatures. Hence, the fluctuations of barrier values take place whereas the barrier $\Delta H=1.4 \mathrm{eV}$ was considered to be constant [33].

It was established that the barrier will be increased with the increase of temperature [34]. Thus, the conclusion was made that the recombinational barrier is determined as the entropy component from the equation (3) [34].

The microscopic model of the entropy barrier was discussed in details in Refs [27, 35]. According to this model, the point defects in silicon (vacancies and self-interstitials) have an extended character at very high tempera- 


\section{V.I. Talanin, I.E. Talanin: Nucleation, growth and transformation of microdefects in FZ-Si}

tures, i.e., one atom (or one vacancy) are extended on some nuclear volumes ( 11 atoms occupied 10 cells). In this model, the recombination happens only for of "simultaneous compression both from these defects in a neighbourhood of one nuclear volume" [34]. The extended defect configurations have the greater number of microstates than a point defect. Thus, the compression lowered an entropy and, therefore, the entropy barrier $\Delta S<0$ exists. If the temperature is lowered the barrier is considerably reduced and disappeared at low temperatures when the defects easily recombine. Thus, the intrinsic point defects at high temperatures are extended, but at low temperatures they have the dumbbell configuration $[34,36]$. The theory of extended defect configurations and the recombinational barrier was confirmed in Refs [37-39].

Thus, according to the theory [34] that was constructed in the correspondence with the Ref. [29] in silicon at high temperatures the joint coexistence of both types of intrinsic point defects in equivalent concentration is observed. This fact determines the values of the self-diffusion factor. Furthermore, "the condition of a smallness" should be taken into account:

$$
\Delta H<<|T \Delta S|
$$

In Ref. [34], using the results of oxidizing experiments and from the value $\exp \left(\Delta G / k_{T}\right)=10^{5}$ the barrier was estimated as $\Delta S=11.5 \mathrm{k}$ at $T=1373 \mathrm{~K}$.

Our accounts according to (3)-(4) give the value of the barrier at $T=1685 \mathrm{~K}$ (temperature of smelting) as $\Delta G=1.674 \mathrm{eV}$.

Hence, the estimate of value from the equation (2) needs to allow the recombinational barrier, i.e.:

$$
\tau \leq \frac{\Omega}{4 \pi D^{S} r_{0} \cdot \exp (-\Delta G / k T)}
$$

The allowance of the barrier value gives the value of $53 \mathrm{~min}$. Thus, for standard sizes of ingots and standard time of its growth the recombination will have no time to come true.

Therefore, we can estimate the length of area near to crystallization front on which the recombination happens according to [19]:

$$
\ell=\frac{2 k T_{m}^{2}}{\left(\Delta H_{i}+\Delta H_{v}\right) G},
$$

where $k$ is the Boltzmann constant; $T_{m}$ is the smelting temperature of; $G$ is the axial temperature gradient; $\Delta H_{i}$ and $\Delta H_{v}$ are energy of self-interstitial and vacancy formation, respectively.

The value of $G$ we can define from the equation (7) with allowance that the gradient is defined on crystallization front:

$G_{L=0}=10+256 \exp (-61.2 \mathrm{~V}-0.28)$
We did account for crystal that was grown at $V=$ $=9 \mathrm{~mm} / \mathrm{min}^{-1}$ when the equality of concentrations of vacancy and interstitial microdefects was established. Thus, from the equations (6) $-(7)$ and at $T_{m}=1685 \mathrm{~K}$, $\Delta H_{i} \approx \Delta H_{v}=4.5 \mathrm{eV}, V=9 \mathrm{~mm} / \mathrm{min}^{-1}$ is established that the $\ell=6.25 \mathrm{~mm}$.

Then the time during which the intrinsic point defects exist in recombinational area is define from the following equation:

$t=\frac{\ell}{V}$,

where $V$ is the growth rate of the crystal. At $V=9 \mathrm{~mm} / \mathrm{min}^{-1}$ is established that $t=41.6 \mathrm{~s}$. The accounts from equations (5)-(8) give at $V=2 \mathrm{~mm} / \mathrm{min}^{-1}$ the following values: $\ell=3.25 \mathrm{~mm}$ and $t=1.625 \mathrm{~min}$. The results [19] show at $V=2 \mathrm{~mm} \mathrm{~min}^{-1}$ the following values: $\ell=2 \mathrm{~mm}, t=1 \mathrm{~min}$ and $\tau \approx 0.3 \mathrm{~s}$.

We received the differences in accounts from the theory [19]. It is possible because of that in Ref. [19] the theory of enthalpy barrier is used and is used that the joint coexistence of both types of intrinsic point defects is denied. According to [36], should exist separately for "interstitial" and separately for "vacancy" of growth regimes, i.e. according to [40]:

$$
\begin{aligned}
& \tau_{i} \approx \frac{1}{4 \pi r D_{i} C_{i} \exp (-\Delta H / k T)} \\
& \tau_{v} \approx \frac{1}{4 \pi r D_{v} C_{v} \exp (-\Delta H / k T)},
\end{aligned}
$$

where $D_{i}$ and $D_{v}$ are diffusivities of a self-interstitials and vacancies respectively; $C_{i}$ and $C_{v}$ are concentrations of self-interstitials and vacancies, respectively.

However, experimental results in Refs [41-47] and our results confirm the fact of joint coexistence of microdefects with various sign of strains and the fact of joint coexistence of intrinsic point defects. These results can be estimated only in the theory of joint coexistence of intrinsic point defects [29] and theory of the entropy barrier [34]. According to the theory [29], in the equation (5) the factor of a joint self-diffusion of vacancies and selfinterstitials is taken into account.

Thus, the experimental results which are in the good correlation with theoretical data allows to approve that at the temperature of smelting in dislocation-free silicon single crystals simultaneous coexistence of equilibrium concentration of vacancies and self-interstitials takes place. Concentration of vacancies and self-interstitials are approximately identical near the crystallization front. The recombination between them in an initial stage of their interaction at a high temperatures is hampered by the entropy barrier. Thus, the disintegration of oversaturation solid solution of intrinsic point defects proceeds simultaneously by two mechanisms: the vacancy and interstitial ones. The microdefects are formed in the result of interaction of intrinsic point defects with impurities of oxygen and carbon. 
For vacancy mechanism, theoretically possible are only vacancy aggregation and joint vacancy-impurities aggregation [19]. Vacancy-impurities aggregation begins earlier than only vacancy aggregation. Interstitial atoms of oxygen are very mobile, and, therefore, the formation of complexes is simulated by a leaving interstitial oxygen in positions of substitution $\mathrm{O}_{s}$ :

$\mathrm{O}_{i}+V_{\mathrm{Si}} \rightarrow \mathrm{O}_{s} \rightarrow 2 \mathrm{O}_{s}+3 \mathrm{O}_{s}+\ldots+{ }_{n} \mathrm{O}_{s}$.

At lower temperatures, $\mathrm{O}_{s}$ can be centres of formation of microprecipitates of oxygen. It is possible to estimate the concentration of oxygen precipitates [20]:

$$
N \approx \frac{C_{v}}{4 \pi \gamma^{2}}\left(\frac{\rho n_{c r}}{\eta}\right)^{1 / 2}\left(\frac{E_{c r}\left|V_{c}\right|}{D C k T^{2}}\right)^{3 / 2},
$$

where $C_{v}$ is the concentration of vacancies; $\rho$ is the density of silicon stices; $n_{c r}$ is the nuclei critical size; $C$ is the concentration of oxygen; $D$ is the diffusivity of oxygen; $E_{c r}$ is the binding energy to one atom of oxygen; $V_{c}$ is the cooling rate.

For FZ-Si at $C_{v} \sim 10^{14} \mathrm{~cm}^{-3}, C \sim 10^{16} \mathrm{~cm}^{-3}, D \sim$ $\sim 10^{-9} \mathrm{~cm}^{2} / \mathrm{s}$ we receive $N \sim 5 \cdot 10^{12} \mathrm{~cm}^{-3}$. Direct TEMresearches of vacancy and interstitial microdefects give the values of their concentration $\sim 10^{13} \mathrm{~cm}^{-3}$.

When microprecipitates are formed, there is a surplus of volume, and one vacancy will be consumed by a growing precipitate for each two oxygen atoms precipitated. The aggregation is accompanied by issue of atoms $I_{\mathrm{Si}}$. The absorption of vacancies and impurity by growing microdefects results in a decreased concentration of vacancies in a comparison with concentration of oxygen. As a result, precipitates begin to absorb oxygen without participation of vacancies, their sizes are increased and then the type of a strain around them varies from vacancy (tensile) to interstitial one (compressive). The similar results were obtained by HREM in Refs [38, 48] The boundary of full transition can be determined from the relation $V / G$. Thus, the parameter $C_{c r i t}$ of the theory [19] does not describe a condition of change of growth regimes ("interstitial" or "vacancy"). This parameter describes conditions of emerging (or vanishing) of microdefects of a vacancy type in the result of diffusion and interaction of point defects during cooling of the crystal. It is possible to determine $C_{c r i t}$ for the critical growth rate (Fig. 2). At $V=$ $=6.25 \mathrm{~mm} / \mathrm{min}^{-1}$ we shall receive $C_{c r i t}=9.31 \cdot 10^{-5} \mathrm{~cm}^{2} /(\mathrm{s} \cdot \mathrm{K})$.

The centers of nucleation based on interstitial oxygen atoms exist also in the case of the interstitial mechanism. However, here the catalytic role is played by carbon atoms. Oversaturation by interstitial silicon atoms results in appearance of complexes $\left[\mathrm{C}_{s} I_{\mathrm{Si}}\right]$ :

$\mathrm{C}_{S}+I_{\mathrm{Si}} \leftrightarrow\left[\mathrm{C}_{s} I_{\mathrm{Si}}\right]$.

The number of interstitial defects can be estimated using the equation [20]:

$$
N \approx 0,13\left(\frac{\rho}{C_{i}}\right)^{1 / 2}\left(\frac{E_{c r}\left|V_{c}\right|}{D_{i} k T^{2}}\right)^{3 / 2},
$$

where $C_{i}$ is the concentration of self-interstitials before condensation; $E_{c r}=1.5 \mathrm{eV}$ is the binding energy for the critical nuclei to one silicon atom.

The estimate for $C_{i} \sim 10^{14} \mathrm{~cm}^{-3}$ and $D_{i} \sim 3.5 \cdot 10^{-4} \mathrm{~cm}^{2} / \mathrm{s}$ gives the value $N \sim 10^{6} \mathrm{~cm}^{-3}$. The similar value is obtained using TEM-researches of $A$-microdefects concentration.

Lowering the critical radius $\left[\mathrm{C}_{S} I_{\mathrm{Si}}\right]$-nucleis and acceleration of a diffusion $\mathrm{C}_{s}$ here happens. As a result, $\left[\mathrm{C}_{S} I_{\mathrm{Si}}\right]$ agglomerates are formed. Furthermore, during supersaturation of $I_{\mathrm{Si}}$ co-precipitation of $\mathrm{O}_{i}$ and $\mathrm{C}_{s}$ can happen $[49,50]$. Thus, for formation of $B$-microdefects:

$I_{\mathrm{Si}}+\mathrm{C}_{s} \rightarrow[C I]+\mathrm{O}_{i} \rightarrow B$-microdefects.

The growth of interstitial microdefects results in significant lowering the self-interstitial concentration. It creates conditions for precipitation of impurities. In this case, formation of particles of an impurity phase is accompanied by generation of self-interstitials in positions between stices of the lattice. As a result, two types of interstitial microdefects are formed: interstitial agglomerates (drains for interstitial atoms of silicon) and impurity precipitates (sources of these atoms).

Both mechanisms (vacancy and interstitial) result in formation of small interstitial agglomerate, i.e. $D$ microdefects. This $D$-microdefects are uniformly distributed $B$-microdefects. The $B$-microdefects is transformed into $A$-microdefects. These mechanisms are described by the following equations.

For the vacancy mechanism:

a) $n \mathrm{O}_{i}+V_{\mathrm{Si}} \rightarrow n\left(V \mathrm{O}_{2}\right) \rightarrow$ vacancy microdefects.

b) $n\left(V \mathrm{O}_{2}\right)+\mathrm{O}_{i}+\ldots+n \mathrm{O}_{i} \rightarrow \mathrm{n}\left[\left(V_{m} \mathrm{O}_{n}\right)+I_{\mathrm{Si}}\right] \rightarrow D-$ microdefects.

For the interstitial mechanism:

a) $\mathrm{C}_{s}+I_{\mathrm{Si}} \rightarrow\left(\mathrm{C}_{s} I_{\mathrm{Si}}\right) \rightarrow D$-microdefects.

b) $\left(\mathrm{C}_{s} I_{\mathrm{Si}}\right)+\mathrm{O}_{i} \rightarrow n\left[\left(\mathrm{C}_{s} I_{\mathrm{Si}}\right)+\mathrm{O}_{i}\right] \rightarrow B$-microdefects.

c) $B$-microdefects $+I_{\mathrm{Si}} \rightarrow A$-microdefects.

In Fig. 3, the scheme of microdefects formation process in FZ-Si is shown.

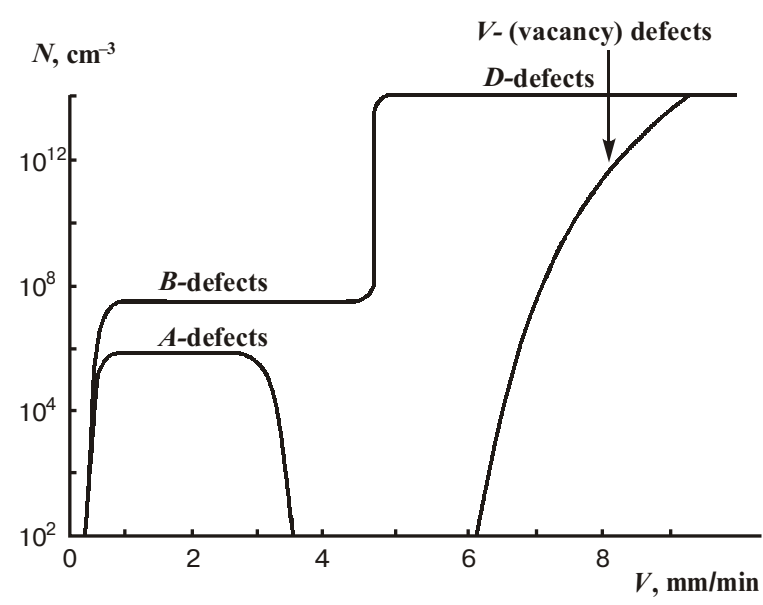

Fig. 3. Schematic diagram of formation and transformation of microdefects in FZ-Si crystals. 


\section{V.I. Talanin, I.E. Talanin: Nucleation, growth and transformation of microdefects in FZ-Si}

\section{Conclusions}

Thus, the formation of vacancy and interstitial microdefects is caused by disintegration of oversaturated solid solution of intrinsic point defects. All the known types of microdefects are formed in the result of interaction of intrinsic point defects with impurities of oxygen and carbon. The concentration of vacancies and self-interstitials are approximately identical in the vicinity of the crystallization front. The recombination between them at the initial stage of their interaction at high temperatures is hampered. Therefore, the formation of microdefects proceeds simultaneously by two mechanisms: the vacancy and interstitial ones. Vacancy and interstitial microdefects coexist in crystal. The parameter $V / G=C_{c r i t}$ describes conditions of emerging (vanishing) of vacancy microdefects in the crystal.

\section{References}

1. Abe T., Samizo T., Maruyama S. // Jpn. J. Appl. Phys., 5(1), P. 255 (1966).

2. Hattori H., Kato N. // J. Phys. Soc. Jpn., 21(9), P. 1773 (1966).

3. Chikawa J., Asada Y., Fujimoto I. // J. Appl. Phys., 41(8), P. 1922 (1970).

4. A.J.R. de Kock // Philips Res. Rept. Suppl., № 1. P. 1 (1973).

5. A.J.R. de Kock, P.J. Roksnoer, P.G.T. Boonen // J. Crystal Growth., 22(4), P. 311 (1974)

6. Foll H., Kolbesen B.O. // J. Appl. Phys., 8(2), P. 319 (1975).

7. Tempelhoff K., Van Sung N. // Phys. Stat. Sol. (a), 70(2), P. 441 (1982).

8. A.A. Sitnikova, L.M. Sorokin, I.E. Talanin et al. // Phys. Stat. Sol. (a)., 81(2), P. 433 (1984).

9. Abe T., Harada H., Chikawa J. // Physica B/C., 116(1), P. 139 (1983).

10. Petroff P.M., De Kock A.J.R. // J. Cryst. Growth., 30(1), P. 117 (1975).

11. N.V. Veselovskaya, E.G. Sheikhet, K.N. Neimark, E.S. Falkevich // In: Rost i legirovanie polyprovodnikovych kristallov i plenok. Novosibirsk: Nauka, 1977. V. 2. P. 284.

12. P.J. Roksnoer, M.M.B. van den Boom // J. Crystal Growth, 53(3), P. 563 (1981).

13. Foll H., Gosele U., Kolbesen B.O. // J. Cryst. Growth, 40(1), P. 90 (1977).

14. Van Vechten J.A. // Phys. Rev. B, 17(10), P. 31971978.

15. Roksnoer P.J. // J. Cryst. Growth, 58(3), P. 596 (1984).

16. Hu S.M. // J. Vac. Sci. \& Technol., 14(1), P. 17 (1977).

17. Sirtl E. // Semiconductor Silicon 1977 (Electrochem. Soc., Pennington, N.Y., 1977) p. 4.

18. De Kock A.J.R. // Defect in Semicond. (North-Holland Publ. Co., Amsterdam, 1981) p. 309.
19. V.V. Voronkov // J. Crystal Growth, 59(3), P. 625 (1982).

20. Voronkov V.V., Milvidskii M.G. // Kristallografiya, 33(2), P. 471 (1988).

21. V.V. Voronkov, R. Falster // J. Crystal Growth, 194(1), P. 76 (1998).

22. Sirtl E., Adler A. // Z. Metalkunde., 52(3) P. 529 (1961).

23. M.F. Ashby, L.M. Brown // Phil. Mag., 8(12), P. 1649 (1963).

24. J.B. Mitchell, W.L. Bell // Acta Met., 24(2), P. 147 (1976).

25. H. Foll, M. Wilkens // Phys. Stat. Sol. (a), 31(4), P. 519 (1975).

26. Frank F.C., Turnbull D. // Phys. Rev., 104(5), P. 617 (1956).

27. Gosele U., Frank W., Seeger A. // J. Appl. Phys., 23(2), P. 361 (1980).

28. Tan T.Y., Gosele U., Morehead F.F. // J. Appl. Phys., 31(1), P. 97 (1983).

29. Morehead F.F., Stolwijk N.A., Meyberg W., Gosele U. // Appl. Phys. Lett., 42(4), P. 690 (1983).

30. Okino T., Onishi M. // Jpn. J. Appl. Phys., 33(11), P. 6642 (1994).

31. Okino T., Shimosaki T., Takaue R. // Jpn. J. Appl. Phys., 36(10), P. 6591 (1997).

32. Tempelhoff K., Van Sung N. // Phys. Stat. Sol. (a), 72(4), P. 617 (1983).

33. Antoniadis D.A., Moskowitz I. // J. Appl. Phys., 53(9), P. 6788 (1982).

34. Gosele U., Frank W., Seeger A. // Sol. State Commun., 45(1), P. 31 (1983).

35. Stolwijk N.A., Holzl J., Frank W. et al. // Phys. Stat. Sol. (a), 104(1), P. 225 (1987).

36. Dzelme J., Ertsinsh I., Zapol B., Misiuk A. // Phys. Stat. Sol. (a), 171(1), P. 197 (1999).

37. Plekhanov P.S., Gosele U., Tan T.Y. // J. Appl. Phys., 84(5), P. 718 (1998).

38. Fedina L., Gutakovskii A., Aseev A. et al. // Phys. Stat. Sol. (a), 171(1), P. 147 (1999)

39. Pizzini S. // Phys. Stat. Sol. (a), 171(1), P. 123 (1999).

40. Waite T.R. // Phys. Rev., 107(3), P. 463 (1957).

41. A.A. Sitnikova, L.M. Sorokin, I.E. Talanin et al. // Phys. Stat. Sol. (a), 90(2), P. K31 (1985).

42. Sitnikova A.A., Sorokin L.M., Talanin I.E. et al. // Fizika Tverdogo Tela, 28(6), P. 1829 (1986).

43. V.T. Bublik, N.M. Zotov // Cryst. Rep., 42(6), P. 1147 (1997).

44. S. Iida, Y. Aoki, K. Okitsu et al. // Jpn. J. Appl. Phys., 37(1), P. 241 (1998).

45. N. Nango, S. Iida, T. Ogawa // J. Appl. Phys., 86(11), P. 6000 (1999).

46. Dornberger E., Von Ammon W., Virbulis J. et al. // J. Cryst. Growth, 230(1), P. 291 (2001).

47. Larsen T.L., Jensen L., Ludge A. et al. // J. Cryst. Growth. 230(1), P. 300 (2001).

48. Fedina L., Gutakovskii A., Aseev A. et. al. // Phil. Mag. A, 77(2), P. 423 (1998).

49. Gupta S., Messoloras S., Schneider J.R. et al. // Semicond. Sci. \& Technol., 7(1), P. 5 (1992).

50. Yamanaka H. // Jpn. J. Appl. Phys., 33(6), P. 3319 (1994). 